Gut, 1988, 29, 1007-1010

Methods and techniques

\title{
Computer aided positioning of manometric probe in the upper digestive tract
}

\author{
M BORTOLOTTI AND G BERSANI \\ From the 1st Medical Clinic, University of Bologna, Italy
}

SUMmaRY A method not needing fluoroscopic control for positioning a manometric probe in the gastroduodenal tract is described. It is based on hydrostatic pressure variations occurring at each recording port of a multichannel perfused probe after changes in patient's position. The data are fed into a computer and the schematic representation of the probe showing its position and direction appear on the CRT screen. The results were visually and mathematically compared with those obtained with fluoroscopy and showed good correspondence between the two techniques in identification of the position of the probe.

The manometric examination of the stomach and duodenum usually requires fluoroscopic control to ensure that the tip of the multichannel probe is directed towards the pylorus and is passed into the duodenum. In fact, with smooth probes and large stomachs the most common occurrence is that the tip of the probe turns up towards the gastric fundus. $X$ ray examination of the abdomen, however, requires time by the radiologist and by the motility examiner to check the probe position, an added cost for the hospital, and a radiation hazard for the patient and the staff. Therefore, we have devised a simple method for determining the position of the gastroduodenal probe that avoids the use of $x$-rays. This is based on the hydrostatic pressure variations that occur after pre-established changes in patient position, at each recording port of the probe. As is known, the pressure recorded at each recording port is the algebraic sum of the intraluminal pressure and the hydrostratic pressure, which is proportional to the vertical distance of the recording hole from a horizontal plane passing at the height of the transducer. In normal conditions, the hydrostatic pressure is negligible if the transducers are positioned at the

Address for correspondence: Dr M Bortolotti, Istituto Clinica Medica e Gastroenterologia dell' Universtita di Bologna, Via Massarenti 9, 40138 Bologna, Italy.

Received for publication 25 January 1988. same level as the recording holes and if the gut examined and the patient maintain the same position until the end of the examination. We took advantage of the effect of hydrostatic pressure variations on the recording ports of the probe, in different body positions, to determine their location in the stomach and duodenum.

\section{Methods}

It is known that to define the position of a point on a plane it is necessary to use the Cartesian coordinates. Similarly the position of each pressure recording point of the probe may be plotted on a Cartesian system of axes lying in a frontal vertical plan passing through the human trunk in vertical position. In this system the abscissa is represented by the crossing line of the frontal plane with an horizontal plane passing through the pressure transducers and the ordinate is a vertical line passing through the xiphisternum and the umbilicus. The distance of each pressure recording point from the abscissa ( $y$ value) and from the ordinate ( $x$ value) is proportional to the hydrostatic pressure measured at the recording point with the trunk in vertical position and in lateral position, respectively. The determination of these Cartesian coordinates is shown by the following two procedures: (1) study in human beings; (2) bench study. 


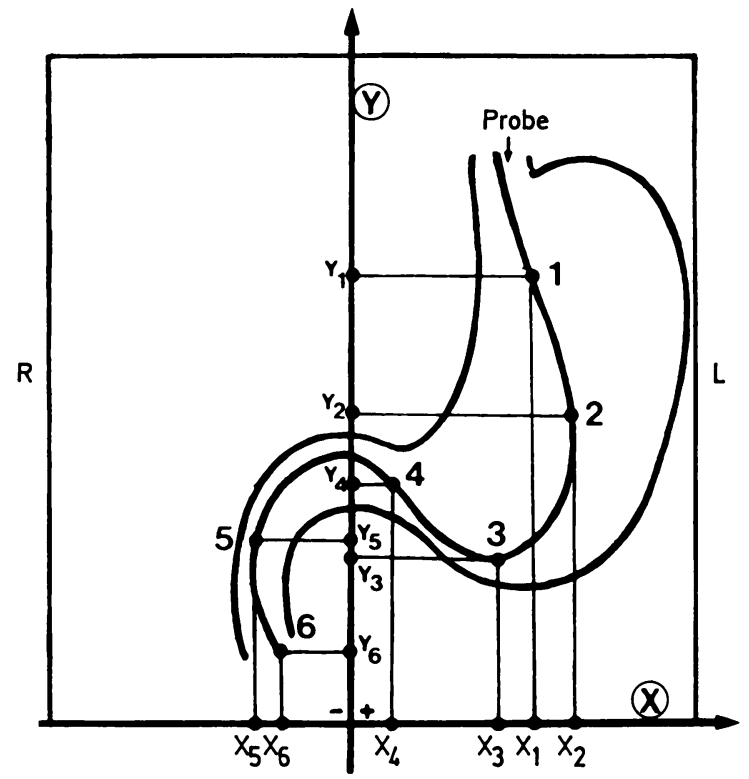

Fig. 1 Cartesian system of axes $(x)$ and $(y)$, where all the hydrostatic value obtained from each pressure recording point of the probe $\left(x_{1}-x_{6}\right)\left(y_{1}-y_{6}\right)$ are plotted. The computer equipped with an appropriate program connects with line segments all these points obtaining on the screen of the monitor a schematic image of the probe as shown in Fig. $2 b$ (III).

In both studies we used the same manometric multichannel probe with six side ports, $5 \mathrm{~cm}$ apart, marked with radioopaque plugs, perfused with a pneumohydraulic low compliance pump, and connected to external pressure transducers Statham P $23 \mathrm{~dB}$ and a polygraph.

The study in human beings was carried out in 10 patients referred for diagnostic upper gastrointestinal tract manometry, which was done just after the probe was positioned in the following manner.

As soon as the patient has been intubated, the multichannel probe is connected to the transducers and the pressures are recorded on the polygraph for a few minutes during each of the following positions of the patient: supine, left lateral decubitus, right lateral decubitus, and sitting up. The patient should be returned to the supine position before each successive change of position. Then the values of pressure variation in the change from the supine position to each of the other three positions are measured on the tracing for each recording port. In this manner each recording port showed three pressure values, which increase as their vertical distance from the horizontal plane of the transducers increases. Each value obtained in the sitting position is called $y$, the values obtained in right $(\mathrm{R})$ and left $(\mathrm{L})$ lateral decubitus are called $x R$ and $x L$ respectively. These data are fed into a microcomputer (Apple II plus) equipped with an appropriate program (listing of which is available on request), that immediately shows on the monitor the schematic representation of the probe in the frontal plane of the patient. Briefly, the computer calculates the algebraic difference between $\mathrm{xR}$ and $\mathrm{xL}$ values, obtaining a value $(x)$ that represents the distance of each recording port from the longitudinal median axis (Fig. 1), then plots on the CRT monitor the points (identified by $\mathrm{x}$ and $\mathrm{y}$ values) for each recording port and connects each point to the following one with line segments obtaining (Fig. 2a) a schematic representation of the probe in the body of the patient. The time taken by the complete procedure is about four minutes.

Subsequently in each patient a radiograph of the upper abdomen (Fig. 2b) was made in order to document the real position of the probe and to compare this image with that obtained on the screen of the monitor.

For the bench study, the manometric probe, curved as indicated in Figure 1 and fixed to a rigid plane which represents the trunk of the patient body, was connected to pneumohydraulic pump, to pressure transducers and to a polygraph. Then the same manoeuvres and calculations, previously described for the patient were carried out for the probe model in order to obtain on the screen of the monitor the schematic image of the probe model.

\section{VALIDATION OF THE METHOD}

\section{Study in patients}

The comparison between the position of the probe shown on the screen of the monitor and the real shape of the probe in the body of the patient, as ascertained by the corresponding radiograms, was carried out (a) by visual inspection, (b) by a mathematical procedure.

The visual comparison between the probe position inferred from the images taken up from the monitor and that observed in the patients radiographs was done by another person blind.

The mathematical comparison between the monitor images and the patients radiographs was carried out as follows: The configuration of the images taken up from the monitor and those taken up from the corresponding radiographs were plotted on a cartesian system of axes, the origin of this (point 'zero') was coincident with the most distal pressure recording port of the probe (Fig. 3). We measured the angles between the line $\mathrm{x}$ and the lines connecting each of the other recording points with the point 'zero' (Fig. 3). As the distance between subsequent recording points is always $5 \mathrm{~cm}$, the orientation of 


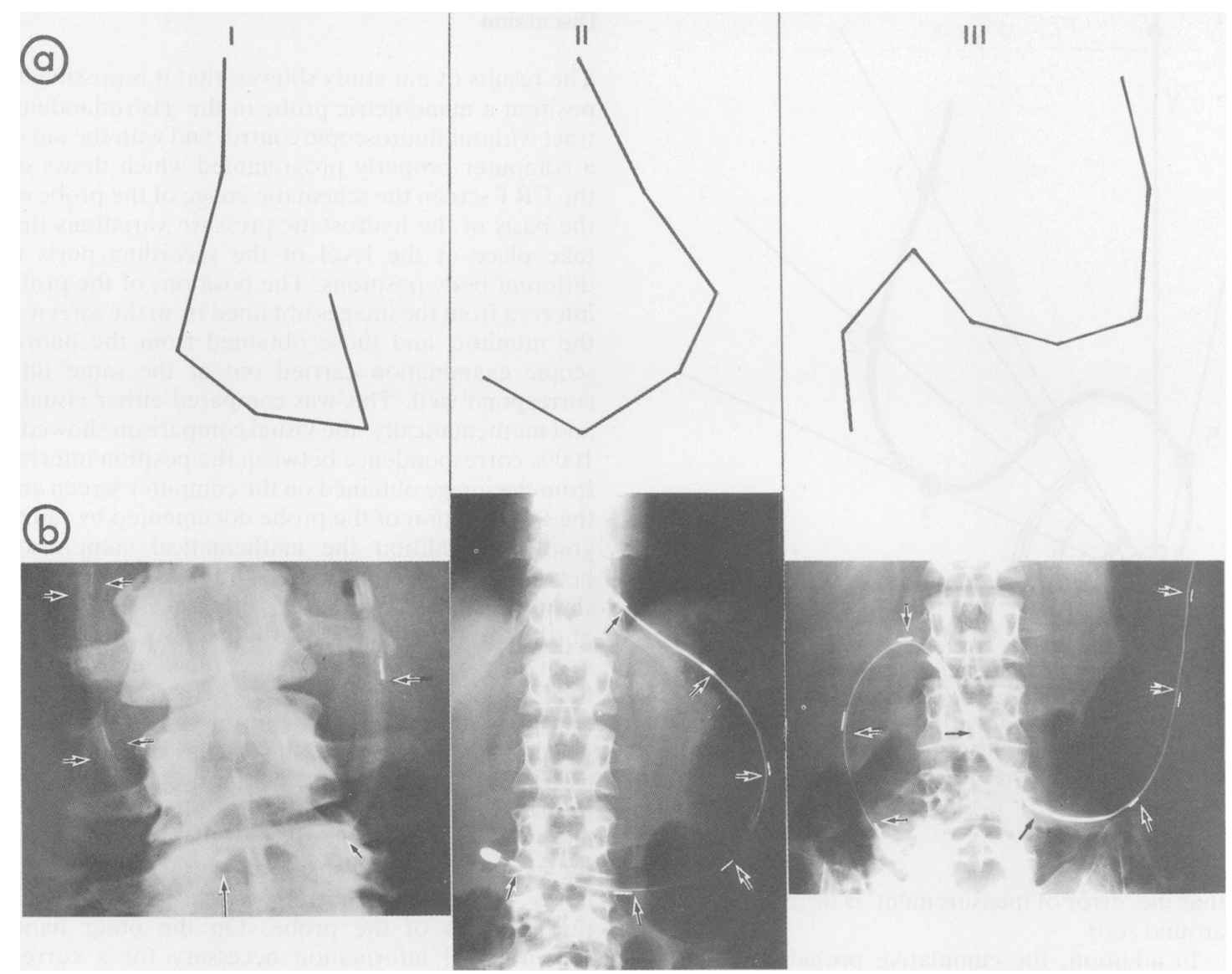

Fig. 2 (a) Series of schematic images of the manometric probe taken up from the screen of the computer and obtained from patients where the probe (i) is directed towards the pylorus, (ii) is passed into the duodenum and (iii) is turned towards the fundus. (b) Corresponding radiographs taken at the same moment. The visual comparison shows a clear correspondence between the radiographs and the computer images of the probe.

the probe in the space with respect to the tip (point 'zero') is defined, in univocal manner, by the sequence and width of the angles calculated as previously. Then we calculated in each case the difference between the angles measured from the radiographic image and the corresponding angles obtained from the screen image. This difference was called 'error of measurement'. We calculated the average of the 'error' with the 95\% interval of confidence and the cumulative probability distribution of 'errors of measurement'.

\section{Bench study}

The procedure described above, with visual and mathematical comparisons, was applied in a similar way on the position inferred from the screen image and the shape of the probe model.

\section{Results}

PATIENTS STUDY

\section{Visual comparison}

On the basis of the computer images it was possible to identify in $100 \%$ of cases the configuration and the position of the probe which was confirmed by radiograms. An example of this is shown in Figure 2 where the probe images obtained from the CRT screen in various positions of the probe are compared with the corresponding radiograms.

\section{Mathematical comparison}

The average of the 'error of measurement' in the 10 cases examined was $4^{\circ}$, with a $95 \%$ interval of confidence of $-14^{\circ}$ and $+6^{\circ}$. Therefore it indicates 


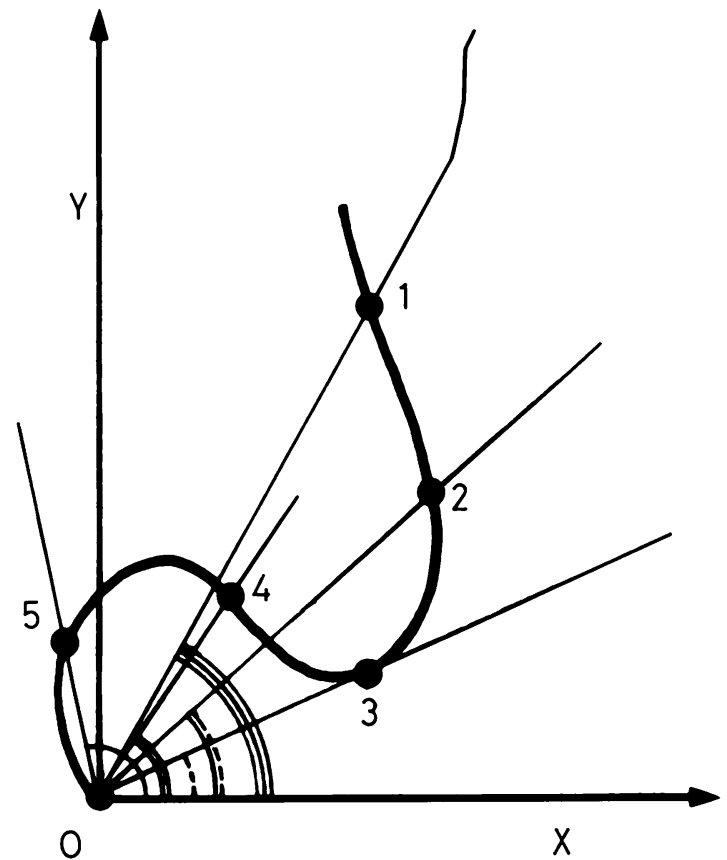

Fig. 3 Method to define mathematically the shape of the probe with respect to the distal recording point (point 'zero'). The orientation of the probe is defined in an univocal manner by the sequence and width of the angles between the line $(x)$ and the lines connecting each of the recording points with the point zero.

that the 'error of measurement' is distributed strictly around zero.

In addition, the cumulative probability distribution of errors indicates that about $80 \%$ of 'errors' are below $20^{\circ}$ which is quite a small interval of variation.

\section{BENCH STUDY}

(a) The visual comparison between the shape of the probe model and the image on the CRT screen gave $100 \%$ of positive results. (b) The mathematical examination showed that $80 \%$ of 'errors of measurement' was below $10^{\circ}$. This result is better than that in patients, and this is most likely because the recording system in the bench study is not affected by external 'noise' that may occur in the patient study.

\section{Discussion}

The results of our study showed that it is possible to position a manometric probe in the gastroduodenal tract without fluoroscopic control and with the aid of a computer properly programmed which draws on the CRT screen the schematic image of the probe on the basis of the hydrostatic pressure variations that take place at the level of the recording ports in different body positions. The positions of the probe inferred from the images obtained from the screen of the monitor, and those obtained from the fluoroscopic examination carried out at the same time correspond well. This was compared either visually and mathematically: the visual comparison showed a $100 \%$ correspondence between the position inferred from the image obtained on the computer screen and the true position of the probe documented by radiogram. In addition the mathematical comparison between our system and the radiological method showed that the geometrical difference in the shape of the probe obtained with the two methods is negligible and does not influence the identification of the position of the probe. These 'errors' may be due to a small differences in the intraluminal basal tone, which may take place during the few minutes of the hydrostatic measurement. Naturally, it is necessary to avoid measurements during phase III of the migrating motor complex. These slight differences in the evaluation of the shape of the probe, however, never prevented a correct conclusion about the real position of the probe. On the other hand, the principal information necessary for a correct positioning of the tip of the probe is as follows: the tip of the probe is in the gastric fundus, the tip is in the distal antrum, the tip is passed in the duodenum. Our method is able to give this kind of information on the screen of the computer. In addition, our method is time and money saving, especially for laboratories that do not have a radiography unit available in the same room where the manometric examination is performed. Moreover, this method does not expose the patient and the physician to a radiation hazard, and we believe that it could replace the traditional $x$ ray method in positioning the manometric probes in the gastroduodenal tract. 